\title{
Hand Plate
}

National Cancer Institute

\section{Source}

National Cancer Institute. Hand Plate. NCI Thesaurus. Code C34185.

The terminal portion of the upper limb buds in an embryo, appearing at around the fifth week of gestation, that give rise to the digits. 